\title{
Norois
}

Environnement, aménagement, société

$235 \mid 2015$

La directive cadre sur l'eau à l'échéance 2015. Les

façades océaniques

\section{Avancées, limites et perspectives de la Directive Cadre sur l'eau à l'échéance 2015}

Improvements, limits and perspectives of the EU-WFD: a 2015 point of view

\section{Grégoire M. Maillet}

\section{(2) OpenEdition}

\section{Journals}

Édition électronique

URL : http://journals.openedition.org/norois/5606

DOI : 10.4000 /norois.5606

ISBN : 978-2-7535-4775-9

ISSN : $1760-8546$

\section{Éditeur}

Presses universitaires de Rennes

\section{Édition imprimée}

Date de publication : 15 novembre 2015

Pagination : $7-13$

ISBN : 978-2-7535-4771-1

ISSN : 0029-182X

Référence électronique

Grégoire M. Maillet, « Avancées, limites et perspectives de la Directive Cadre sur l'eau à l'échéance 2015 », Norois [En ligne], 235 | 2015, mis en ligne le 15 novembre 2015, consulté le 22 septembre 2020. URL : http://journals.openedition.org/norois/5606 ; DOI : https://doi.org/10.4000/norois.5606 


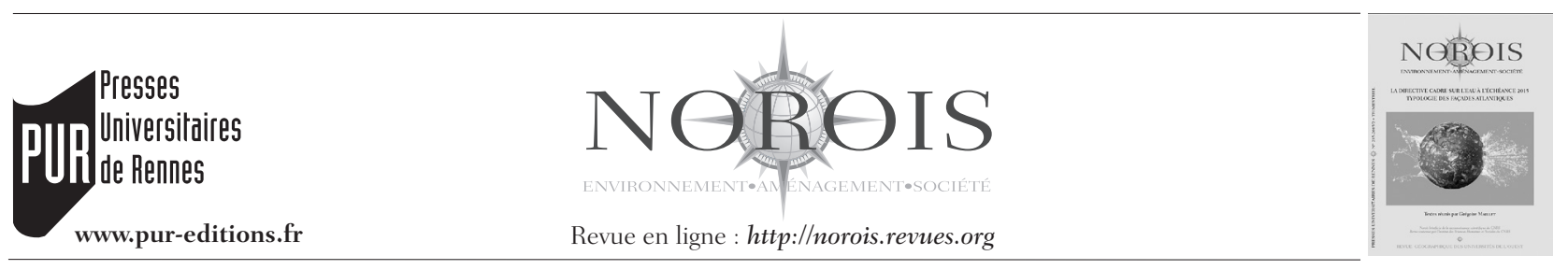

\title{
Introduction \\ Avancées, limites et perspectives de la Directive Cadre sur l'Eau à l'échéance 2015
}

\author{
Grégoire M. MaILlet
}

LPG-BIAF, UMR 6112 CNRS, Université d'Angers, Faculté des Sciences - 2 Bd Lavoisier, 49045 Angers cedex 01, France. (gregoire.maillet@univ-angers.fr)

\section{INTRODUCTION : LA DCE À L'HORIZON 2015}

La Directive Cadre sur l'Eau (European Commission, 2000) a pour but de promouvoir une utilisation durable (tant qualitative que quantitative) de l'eau et, pour ce faire, s'applique aux 27 États de l'Union Européenne depuis l'an 2000. L'année 2015 est une limite charnière puisque l'objectif fixé était d'atteindre un bon état chimique et écologique de toutes les eaux européennes pour cette année. Le bilan d'aujourd'hui force à multiplier les constats d'échec (M.A.P., 2008; EEA, 2012) face à la forte résilience des pollutions, aux trajectoires évolutives tenaces des écosystèmes et à l'inertie des pratiques sociétales et des systèmes de production (agricoles notamment) (Folke et al., 2002 ; T. Pointet, 2005; Moreno-Mateos et al., 2012; P. Geoff, 2014). Cette conjoncture a conduit à considérer que, dans les situations où cet objectif ne pouvait être atteint en 2015, des extensions pourraient être accordées jusqu'à l'échéance limite de la Directive fixée à 2027, sous réserve qu'elles soient demandées et justifiées.

De fait, si nombre de masses d'eau sont effectivement « en bon état » actuellement (figure 1 - planche I), rares sont celles qui ne l'étaient pas et qui le sont devenues sous l'impulsion de la DCE (Ioris, 2015; Kanakoudis et al., 2015).

\section{BASES STRUCTURELLES ET SCIENTIFIQUES DE LA DCE}

Pourtant la Directive a bénéficié pour sa rédaction du retour d'expérience de l'approche par le modèle DPSIR (FPEIR en français pour Forces motricesPressions-Etat-Impact-Réponses), approche globale formalisée par l'OCDE (OCDE, 1991; OCDE, 1998) visant à décrire les interactions entre la société et l'environnement à l'aide d'indicateurs et de statistiques. Ce transfert d'expérience s'est concrétisé dans la Directive par la reprise à son compte des concepts de « gestion intégrée », d' "état de référence », ou de «bon état des masses d'eau », éléments novateurs et gages théoriques de la réussite de cette première tentative de définition d'un cadre européen pour la politique de l'eau (Loupsans, 2013).

Qui plus est, force est de constater que la transposition de cette directive en France a été particulièrement bien prise en charge et d'application rapide 
(loi 2004.338 du 21 avril 2004). Le positionnement en première ligne des Agences de bassins hydrographiques, puis la (re)création de l'ONEMA par la loi sur l'eau et les milieux aquatiques (Lema) du 30 décembre 2006, ont en effet permis une mise en œuvre et un accompagnement aussi pertinents que possible des différentes étapes d'application de la Directive au travers des SDAGE (délivrance d'un état des lieux, identification des verrous méthodologiques, définition des connaissances manquantes, rédaction de cahier des charges pour la mise en place de mesures correctives ou compensatoires, évaluation économique de la faisabilité de ces mesures, consultations publiques, planification d'actions, communication...) (Barraud et Germaine, 2013).

\section{Causes des limites identifiées}

Les raisons de ce relatif échec ne sont donc pas à rechercher dans l'application de la méthode mais plus probablement dans les limites du cahier des charges initial, vraisemblablement encore immature au moment de la publication de la Directive. En effet, il y avait entre 1994 et 2000, période d'écriture de la Directive, un décalage entre la prise de conscience du niveau alarmant de la qualité des eaux européennes, les besoins des financeurs public en matière de production d'indicateurs quantitatifs, et les attentes légitimes des politiques en termes de délais, d'un côté et, d'un autre côté, les connaissances scientifiques nécessaires ne serait-ce qu’à l'élaboration des protocoles d'évaluation. Mais c'est sans doute aussi la capacité des hydrosystèmes à exprimer les fruits d'opérations de réparation/restauration/compensation qui aurait été largement sous-estimée (J.-C. Dauvin, 2007 ; Bouleau et Pont, 2014).

Il semble, de fait, que le fossé se soit progressivement creusé entre un monde de gestionnaires s'appliquant à rendre la DCE aussi efficace que possible, et un monde académique, d'abord lent à réagir (Lévêque, 2013), puis s'orientant essentiellement à rebours vers la redéfinition des concepts de base ou la validation/perfectionnement/reformulation de protocoles d'études. Ainsi, sans s'opposer aux agences de l'État et aux bureaux d'étude, la communauté scientifique s'est essentiellement évertuée au cours des 10 dernières années à repérer les lacunes et incohérences de la DCE. À titre d'exemple, on soulèvera la dénonciation de la prise en compte tardive des effets de la variabilité hydroclimatique à court ou long terme sur les hydrosystèmes (D. Pont, 2006; Lespez et al., 2013; Beniston et al., 2014; Lalubie et al., ce volume) ou sa non influence dans les processus de prise de décision (Kiparsky et al., 2012). On notera aussi les remarques sur la délicate application de la Directive dans les espaces transfrontaliers (Maury et Richard, 2011 ; Da Costa Silva et al., 2013; Dave et Munawar, 2014) ou sur le peu d'empressement à mettre en œuvre la dimension participative que la Directive appelait pourtant de ses vœux (Steyaert et Ollivier, 2007).

Mais le point central de divergence réside surtout dans la remise en cause par les chercheurs des principes fondateurs de la DCE L'identification, la délimitation et la définition des masses d'eau de l'Union Européenne est remise en cause (Dauvin, 2011 ; Bartout, ce volume). Le concept de «gestion intégrée » des bassins versants fait très tôt l'objet d'objections de fond (Ruf et Valony, 2007; Linton et Brooks, 2011; O. Graefe, 2013; Lajeunesse et al., ce volume). Celui d'«état de référence » fait, quand à lui, l'objet de controverses plus techniques (Stoddard, 2006; Dufour et Piégay, 2009; Bouleau et Pont, 2015; Lalubie et al., ce volume) car il présuppose la définition de valeurs de référence qui renvoient la plupart du temps à une définition à dires d'expert basée sur des séries historiques décennales ( 1990-2000) incomplètes ou non-homogènes (Buchet, 2012). Plus fondamentalement, ce concept s'oppose au principe de «trajectoire évolutive » (Lévêque, 2013) dans une vision un peu conservationniste voire fixiste que dénonçait déjà Colinvaux en 1982. Quant à la notion de «bon état écologique », structurellement liée à un état de référence «forcément » faiblement anthropisé (Roche et al., 2005), elle est fortement critiquée par la communauté scientifique en raison, notamment, de la subjectivité d'une évaluation simpliste de l'écosystème, basée exclusivement sur la qualité des eaux de surface (Barraud et Germaine, 2013).

Ainsi, à l'échéance 2015, alors que les gestionnaires ont fait le point (Dupont-Kerlan, 2014) et que les décideurs tirent des bilans (European Commission, 2015), les scientifiques en sont encore à roder les indicateurs, tant géochimiques (e.g. Nowell, 2014; Lajeunesse et al., ce volume) que biologiques (e.g. Ducrotoy et al., 2011 ; Feio et 
al., 2014); tandis que l'évaluation des impacts économiques (analyses coût/bénéfices) n'en est encore qu'au stade de l'élaboration des protocoles pluridisciplinaires (écologie-économie) nécessaires aux calculs (Martin-Ortega et al., 2015). Sans parler des toujours persistantes difficultés d'homogénéisation de ces protocoles entre les partenaires européens (Carletti et Heiskanen, 2009; Pardo et al., 2012; Birk et al., 2013; Poikane et al., 2014, Poikane et al., 2015)...

\section{Plus-value de Ce dossier spécial}

L'objectif de ce dossier spécial DCE, qui se voulait plus exhaustif, est d'illustrer selon des approches disciplinaires très distinctes, le regard que des spécialistes, gestionnaires, chercheurs ou agents de l'État confrontés à la mise en ouvre de la DCE, portent sur leur domaine d'expertise en 2015. Ils ont vocation à produire des éclairages sur différentes étapes de la mise en œuvre de la DCE, tout en identifiant les contours de futurs débats (restauration, appropriation citoyenne) au-delà même du nouveau terme prévu de la directive (en 2027).

Ainsi, P. Bartout initie la réflexion en tentant d'évaluer la qualité de la perception qu'à l'Europe de ses propres masses d'eau. La masse d'eau, découpage territorial élémentaire des milieux aquatiques destinée à être l'unité d'évaluation de la DCE, est en effet un objet qui demande à être caractérisé avec précision, afin, entre autres, que les protocoles de caractérisation et de suivi de l'état écologique soient adaptés à la diversité des configurations. En s'appuyant sur l'exemple des plans d'eau, l'auteur démontre la mise à l'écart «en partie subie, en partie voulue » de près de 99,8 \% des plans d'eau européens. Il pointe ainsi, outre une méconnaissance criante du territoire de l'Union européenne, un biais significatif dans l'orientation des politiques publiques et des priorités fixées aux états membres.

O. Le Pape, M. Lepage, et P. Féra, quant à eux, reviennent sur la diversité des masses d'eau et le décalage de connaissance qui existait entre ces milieux. Ils illustrent le bilan DCE par un retour sur les avancées méthodologiques qu'aura permises la Directive dans les milieux estuariens. En replaçant les éléments dans leur contexte historique, ils explicitent le contexte de déficit de connaissances scientifiques et méthodologiques auquel les Agences de bassin ont du faire face dans la mise en œuvre de la Directive. Puis ils exposent les différentes étapes franchies au pas de charge pour parvenir à proposer en 2015 des protocoles efficaces, tant en matière de bio-indicateurs pertinents pour les masses d'eau de transition, qu'en termes de collaboration aboutie pour espérer une réelle gestion intégrée des estuaires et eaux côtières.

Un autre aspect de la mise en œuvre de la DCE est traité par l'article de G. Lalubie, J.-R. GrosDésormeaux, et T. Nicolas. En dénonçant la minimisation de l'impact du changement climatique sur la qualité des eaux aux Antilles françaises, ils touchent du doigt les conséquences d'une sous-estimation de l'hétérogénéité du territoire d'application de la DCE et de la trop contraignante normalisation des méthodes et temporalités qui en résultent. Ceci se traduit, dans leur cas d'étude, par une forte limitation dans la capacité des DROM à caractériser un état de référence fiable de leurs masses d'eau, un moindre leadership des agences de l'état par rapport à la métropole, et un plus délicat découplage entre les objectifs DCE, l'institutionnalisation du principe de précaution et la réduction des vulnérabilités climatiques de ces territoires. Ils concluent en proposant un réel effort d'implication de l'ensemble des acteurs de l'environnement dans une configuration où les moyens financiers alloués seront à la hauteur des verrous à dépasser et des synergies à développer.

Pour clore ce dossier, I. La Jeunesse et ses collaborateurs testent l'efficacité de la mise en œuvre d'un plan de gestion de bassin-versant visant à réduire la contamination d'un cours d'eau. Ce n'est certes qu'un exemple parmi d'autres, mais il permettra au lecteur de se faire une idée de la réalité des situations auxquelles les gestionnaires sont confrontés, et de l'efficacité des injonctions européennes sur la restauration des hydrosystèmes. Au-delà de cet objectif, cet article est une illustration de travail collaboratif efficace entre chercheurs, gestionnaires et décideurs/ financeurs, menant à une caractérisation précise de la rémanence des pollutions et à la juste évaluation des objectifs atteignables à l'échéance 2018 .

\section{Perspectives et champs DE RECHERCHE}

Rester au niveau du constat ne peut pas faire évoluer les choses positivement. Très tôt, le risque 
de ne pas atteindre les objectifs en 2015 avait été évalué et modélisé (Borja et al., 2006; Breen et al., 2012). Cette anticipation a permis à de nombreuses composantes du tissu scientifique et managérial de l'environnement de se structurer pour :

- rechercher le bénéfice scientifique qu'il était possible de tirer des études mises en œuvres dans le cadre de la Directive (Dufresne et Flamand, 2009; Argillier et Lepage, 2010; Le Pape et al., ce volume);

- se faire force de proposition avec une véritable volonté de perfectionnement des méthodes et approches, pour que la DCE débouche à l'horizon 2021 sur des résultats plus positifs (WFD CIS, 2010; Hering et al., 2013; Reyjol et al., 2014; Prat et al, 2014 ; Boero et al., 2015; Garnier et al., 2015).

La voie empruntée par la directive-cadre sur la stratégie marine (DCSMM), adoptée par l'Union Européenne en 2008, semble bénéficier à son tour de ce retour d'expérience de la DCE. En prônant de dépasser le simple recours aux descripteurs simplistes (teneurs en contaminants, nombre d'espèces présentes, etc.), elle s'attache de manière pertinente à la compréhension des interactions entre les différents facteurs environnementaux et les rétro-actions entre environnements et écosystèmes. C'est, semble t-il, l'approche que les services en charge de l'application de la DCE devront emprunter sur ce nouveau cycle qui s'ouvre avec l'année 2016 pour espérer des résultats probants à l'horizon 2021.

Certes, les défis scientifiques ne manquent pas sur cette voie qui reste à tracer (Roche et al., 2005; Arnaud-Fassetta et al., 2013). Mais ce processus de parangonnage, encore inachevé en 2015, aura tout de même permis l'émergence de notions fondamentales comme celle de "Alternative State Theory " (Scheffer et al., 2001), de "services rendus par les écosystèmes » (de Groot et al., 2002; Wallace, 2007), ou de «écologisation » (Napoléone et Allaoua, 2014), qui ont ouvert les champs de la recherche en matière d'évaluation des vulnérabilités significatives des écosystèmes, d'amélioration de la qualité des masses d'eau, de restauration des conditions d'équilibre des hydrosystèmes ou de justification de nouveaux dispositifs de consultation du public (Dufour et Piégay, 2009; Chevassus-Au-Louis et al., 2009; Notte et Salles, 2011 ; Maresca et al., 2011 ; Verdonschot et al., 2013). Concepts qui, bien que très prometteurs en matière d'avancées scien- tifiques, de satisfactions intellectuelles et surtout de résultats durables, atteignent déjà de premières limites (Barnaud et al., 2011) et appellent déjà à de nouvelles approches qui, par exemple, tenteraient d'introduire la "convivialité » (Dilling et Lemos, 2011) dans les nécessaires échanges itératifs entre scientifiques et décideurs. C'est par cette refonte totale des relations entre acteurs de la gestion environnementale, et grâce à ces avancées interdisciplinaires qu'aura permises la DCE, que l'on pourra espérer aboutir à la concertation et la participation active de toutes les parties prenantes que la bonne gestion des masses d'eau européenne nécessite.

Le processus de maturation scientifique semble donc en tout point en voie d'achèvement. Il aura nécessité 15 ans mais devrait déboucher sur un cahier des charges applicable et pertinent à l'échelle européenne, et adapté à la grande diversité des masses d'eau. Certains objecteront que l'urgence de la situation aurait exigé une mise en place plus rapide. On peut, de fait, et sans être fataliste, le regretter. Mais ainsi va la Science (et souvent fort heureusement...)!

\section{Bibliographie}

Argillier C., Lepage M., 2010. Que peut-on attendre de la directive cadre européenne sur l'eau en matière de connaissance sur la biodiversité?, Sciences Eaux \& Territoires, 3, p. 84-87.

Arnaud-Fassetta G., Masson E., Reynard E. (eds.), 2013. European continental hydrosystems under changing water policyg, München, Friedrich Pfeil Verla, 344 p.

Barnaud C., Antona M., Marzin J., 2011. Vers une mise en débat des incertitudes associées à la notion de service écosystémique, VertigO - la revue électronique en sciences de l'environnement, 11 (1) [En ligne] : [http://vertigo.revues. org/10905].

Barraud R., Germaine M.-A., 2013. Defining and achieving good water status: expert rule versus local participation. Case studies on dam removal in western France, in Arnaud-Fassetta G., Masson E., Reynard E. (eds.), European continental hydrosystems under changing water policy, Friedrich Pfeil Verlag, München, p. 233-245.

Beniston M., Stoffel M., Hill Clarvis M., Quevauviller P., 2014. Assessing climate change impacts on the quantity of water in Alpine regions: Foreword to the adaptation and policy implications of the EU/FP7 "ACQWA" project, Environmental Sciences \& Policy, 43, p. 1-4.

Birk S., Willby N.J., Kelly M.G., Bonne W., Borja A., PoiKANE S., VAAN DE Bund W., 2013. Intercalibrating classifi- 
cations of ecological status: Europe's quest for common management objectives for aquatic ecosystems, Science of The Total Environment, 454-455C, p. 490-499.

Boero F., Dupont S., Thorndyke M., 2015. Make new friends, but keep the old: towards a transdisciplinary and balanced strategy to evaluate Good Environmental Status, Journal of the Marine Biological Association of the United Kingdom, 95 (6), p. 1069-1070.

Bouleau G., Pont D., 2014. Les conditions de référence de la directive cadre européenne sur l'eau face à la dynamique des hydrosystèmes et des usages, Natures Sciences Sociétés, 22, p. 3-14.

Bouleau G., Pont D., 2015. Did you say reference conditions? Ecological and socio-economic perspectives on the European Water Framework Directive, Environmental Science E Policy, 47, p. 32-41.

Breen P., Robinson L.A., Rogers S.I., Knights A.M., Piet G., Churlova T., Margonski P., Papadopoulou N., Akoglu E., Eriksson A., Finenko Z., Fleming-Lehtinen V., Galil B., Goodsir F., Goren M., Kryvenko O., Leppanen J.M., Markantonatou V., Moncheva S., Oguz T., Paltriguera L., Stefanova K., Timofte F., Thomsen F., 2012. An environmental assessment of risk in achieving good environmental status to support regional prioritisation of management in Europe, Marine Policy, 36 (5), p. 1033-1043.

Buchet R., 2012. Assistance à la coordination des travaux européens d'intercalibration des indicateurs biologiques de la Directive Cadre sur l'Eau (DCE), Rapport Contrat $\mathrm{N}^{\circ}$ 11/5210818 HOCER/IFREMER, $152 \mathrm{p}$.

Carletti A., Heiskanen A.S., 2009. Water Framework Directive intercalibration technical report. Part 3: Coastal and Transitional waters, JRC Scientific and Technical Reports, 244 p.

Chevassus-Au-Louis B., Salles J.-M., Pujol J.-L., 2009. Approche économique de la biodiversité et des services liés aux écosystèmes - Contribution à la décision publique, Paris, Centre d'analyse stratégique/La Documentation Française, $376 \mathrm{p}$.

Colinvaux P., 1982. Invitation à la science de l'écologie, Paris, Seuil, coll. « Points Sciences », 249 p.

Da Costa Silva F., Marques A., Arnaud-Fassetta G., Alonso J., Martins I., Guerra C., 2013. Methodological deepening for flood-risk analysis in cross-border regions: a case study for the Lima catchment (NW Spain/Portugal) according to the implementation of the European Water Framework Directive, in Arnaud-Fassetta G., Masson E., Reynard E. (eds.), European continental hydrosystems under changing water policy, Friedrich Pfeil Verlag, München, p. 99-112.

Dauvin J.-C., 2011. Perception sectorielle face à la nécessité d'une vision globale et partagée de l'estuaire de la Seine, VertigO - la revue électronique en sciences de l'environnement, Hors série 10 : Fleuves, estuaires et cours d'eau : représentations et pratiques, [En ligne] : [http://vertigo. revues.org/11436].

Dauvin J.-C., 2007. Paradox of estuarine quality : benthic indicators and indices or debate for the future, Marine Pollution Bulletin, 55, p. 271-281.
Dave G., Munawar M., 2014. Aquatic ecosystems across boundaries: Significance of international agreements and cooperation, Aquatic Ecosystem Health \& Management, 17 (4), p. 437-446.

de Groot R.S., Wilson M.A., Boumans R.M.J., 2002. A typology for the classification, description and valuation of ecosystem functions, goods and services, Ecological Economics, 41 (3), p. 393-408.

Dilling L., Lemos M.C., 2011. Creating usable science: opportunities and constraints for climate knowledge use and their implications for science policy, Global Environmental Change, 21 (2), p. 680-689.

Ducrotoy J.-P., Mazik K., Elliot M., 2011. Bio-sedimentary indicators for estuaries: a critical reviews, Paris, Édition Union des Océanographes de France, 110 p.

Dufour S., PiÉgay H., 2009. From the myth of a lost paradise to targeted river restoration: Forget natural references and focus on human benefits, River Research and Applications, 25, 5, p. 568-581.

Dufresne S., Flamand L., 2009. La Directive Cadre Européenne sur L'Eau comme support au développement de projets de territoires, Cahier du Littoral, n 19, 12 p.

Dupont-Kerlan E. (dir.), 2014. Programmes de surveillance: des exigences de la directive cadre sur l'eau à la mise en œuvre et à l'utilisation des résultats, Les rencontres de l'ONEMA, 27, 4 p.

EEA (European Environment Agency) 2012. European waters - assessment of status and pressures. Report $n^{\circ}$ 8/2012, 96 p. [En Ligne] : [http://www.eea.europa.eu/publications/european-waters-assessment-2012].

European Commission, 2000. Directive 2000/60/EC of the European Parliament and of the Council of 23 October 2000 establishing a framework for Community action in the field of water policy, Official Journal 2000; L 327, p. 1-72.

European Commission, 2015. Report on the progress in implementation of the Water Framework Directive Programmes of Measures, 4th WFD implementation report COM(2015) 120 final, $143 \mathrm{p}$.

Feio M.J., Aguiar F.C., Almeida S.F.P., Ferreira J., Ferreira M.T., Elias C., Serra S.R.Q., Buffagni A., Cambra J., Chauvin C., Delmas F., Dörflinger G., Erba S., Flor N., Ferréol M., Germ M., Mancini L., Manolaki P., Marcheggiani S., Minciardi M.R., Munné A., Papastergiadou E., Prat N., Puccinelli C., Rosebery J., Sabater S., Ciadamidaro S., Tornés E., Tziortzis I., Urbanic G., Vieira C., 2014. Least Disturbed Condition for European Mediterranean rivers, Science of the Total Environment, 476, p. 745-756.

Folke C., Carpenter S., Elmqvist T., Gunderson L., HolLing C.S., WAlker B., 2002. Resilience and sustainable development: building adaptive capacity in a world of transformations, Ambio, 31 (5), p. 437-440.

Garnier, M., Harper D.M., Blaskovicova L., 2015. Climate Change and European Water Bodies, a Review of Existing Gaps and Future Research Needs: Findings of the ClimateWater Project, Environmental Management, 56 (2), p. 271-285. 
Geoff P., 2014. Progress towards the implementation of the European Water Framework Directive (2000-2012), Aquatic Ecosystem Health \& Management, 17 (4), p. 424-436.

Graefe O., 2013. The river basin as a territorial water management unit - Towards post-political water management, in Arnaud-Fassetta G., Masson E., Reynard E. (eds.), European continental hydrosystems under changing water policy, Friedrich Pfeil Verlag, München, p. 11-16.

Hering D., Borja A., Carvalho L., Feld C.K., 2013. Assessment and recovery of European water bodies: Key messages from the WISER project, Hydrobiologia, 704(1), p.1-9.

Ionis A. A. R., 2015. The prospects for the water management framework in the Douro, Portugal, European Urban and Regional Studies, 22(3), p. 316-328.

Kanakoudis V., Tsitsifli S., Azariadi T., 2015. Overview of the River Basin Management Plans Developed in Greece Under the Context of the Water Framework Directive 2000/60/ EC Focusing on the Economic Analysis, Water Resources Management, 29 (9), p. 3149-3174.

Kiparsky M., Milman A., Vicuña S., 2012. Climate and water: knowledge of impacts to action on adaptation, Annual Review of Environment and Resources, 37, p. 163-194.

Lespez L., Viel V., Cador J-M., Germaine M-A., GermainVallee C., Rollet A-J., Delahaye D., 2013. Environmental dynamics of small rivers in Normandy (western France) since the Neolithic era. What lessons for today in the context of the European Water Framework Directive?, in Arnaud-Fassetta G., Masson E., Reynard E. (eds.), European continental hydrosystems under changing water policy, Friedrich Pfeil Verlag, München, p. 71-86.

LÉVÊQUE C., 2013. L'écologie est-elle encore scientifique?, Paris, Quae éditions, coll. «Essais », 144 p.

Linton J., Brooks D.B., 2011. Governance of transboundary aquifers: new challenges and new opportunities, Water International, 36 (5), p. 606-618.

Lisa H. Nowell J., Norman E., Moran P.W., Martin J.D., Stone W.W., 2014. Pesticide Toxicity Index-A tool for assessing potential toxicity of pesticide mixtures to freshwater aquatic organisms, Science of the Total Environment, 476, p. 144-157.

Loupsans D., 2013. L'influence normative de l'OCDE sur la Directive-cadre européenne sur l'eau (DCE). VertigO - la revue électronique en sciences de l'environnement, 13 (3), [En ligne] : [http://vertigo.revues.org/14377].

MAP, 2008. Plan ECOPHYTO 2018 de réduction des usages de pesticides 2008-2018. Rapport du Ministère de l'Agriculture et de la Pêche (MAP), 19 p. [En ligne] : [http://agriculture. gouv.fr/telecharger-le-plan-ecophyto].

Maresca B., Mordret X., Ughetto A.-L., Blancher P., 2011. Évaluation des services rendus par les écosystèmes en France, Développement durable et territoires, 2 (3) [En ligne]: [http://developpementdurable.revues.org/9053].

Martin-Ortega J., Perni A., Jackson-Blake L., Balana B.B. Mckee A., Dunn S., Helliwell R., Psaltopoulos D., Skuras D., Cooksley S., Slee B., 2015. A transdisciplinary approach to the economic analysis of the European Water Framework Directive, Ecological Economics, 116, p. 34-45.
Maury C., Richard S., 2011 . La difficile gestion de l'eau en contexte transfrontalier : un exemple franco-espagnol, Articulo - Journal of Urban Research, 6, [En ligne] : [http:// articulo.revues.org/1736].

Moreno-Mateos D., Power M.E., Comin F.A., Yockteng R., 2012. Structural and functional loss in restored wetland ecosystems, PLoS. Biology, 10 (1) : e1001247. doi:10.1371/ journal.pbio. 1001247.

Napoléone C., Allaoua A.K. (coord.), 2014. Ecologisation des politiques publiques et des pratiques agricoles, Dossiers de l'environnement de l'Inra, 34, Paris, $154 \mathrm{p}$

Notte O., Salles D., 2011. La prise à témoin du public dans la politique de l'eau. La consultation directive-cadre européenne sur l'eau en Adour-Garonne, Politique Européenne, 33, p. 37-62.

OCDE, 1991. Environmental Indicators: A preliminary set, Paris, 38 p.

OCDE, 1998. Towards Sustainable Development: Environmental Indicators, Paris, $132 \mathrm{p}$.

Pardo I., Gómez-Rodríguez C., Wasson J.-G., Owen R., van de Bund W., Kelly M., Bennett C., Birk S., Buffagni A., Erba S., Mengin N., Murray-Bligh J., Ofenböeck G., 2012. The European reference condition concept: A scientific and technical approach to identify minimally-impacted river ecosystems, Science of the Total Environment, 420, p. 33-42.

Poikane S., Zampoukas N., Borja A., Davies S.P., Van De Bund W., Birk S., 2014 Intercalibration of aquatic ecological assessment methods in the European Union: Lessons learned and way forward, Environmental Science \& Policy, 44, p. 237-246.

Poikane S., Birk S., Boehmer J. et al., 2015. A hitchhiker's guide to European lake ecological assessment and intercalibration, Ecological Indicators, 52, p. 533-544.

Pointet T., 2005. Les mesures concrètes pour la mise en œuvre de la Directive Cadre européenne sur l'Eau (DCE) : quels chantiers d'ici 2015?, Géosciences, 2, p. 6-13.

Pont D. (éd.), 2006. Conséquences du changement global sur la distribution et la démographie du Chabot (Cottus gobio) : une approche intégrative, Actes du Colloque de restitution IFB - GICC Réponses adaptatives au changement global : résultats et prospective, 18-20 septembre 2006, Paris, p. 28-36.

Prat N., Gallart F., Von Schiller D., Polesello S., GarCía-Roger E.M., Latron J., Rieradevall M., Llorens P., Barberá G.G., Brito D., De Girolamo A.M., Dieter D., Lo Porto A., Buffagni A., Erba S., Nikolaidis N.P., Querner E.P., Tournoud M.G., Tzoraki O., Skoulikidis N., Gómez R., Sánchez-Montoya M.M., Tockner K., Froebrich J. , 2014. The Mirage toolbox: an integrated assessment tool for temporary streams, River Research and Applications, 30, p. 1318-1334.

Reyjol Y., Argillier C., Bonne W., Borja A., Buijse A.D., Cardoso A.C., Daufresne M., Kernan M., Ferreira M.T., Poikane S., Prat N., Solheim A.L., Stroffek S., Usseglio-Polatera P., Villeneuve B., van de Bund W., 2014. Assessing the ecological status in the context of the European 
Water Framework Directive: Where do we go now?, Science of The Total Environment, 497-498C, p. 332-344.

Roche P.-A., Billen G., Bravard J.-P., Décamps H., PenneQuin D., Vindimian E, Wasson J.-G., 2005. Les enjeux de recherche liés à la directive-cadre européenne sur l'eau, $C$. R. Geoscience 337, p. 243-267.

Ruf T., Valony M.-J., 2007. The contradictions in the integrated management of water resources in irrigated Mediterranean agriculture. Cahiers Agricultures, 16 (4), p. 294-300.

Scheffer M., Carpenter S., Foley J.A., Folke C., Walker B., 2001. Catastrophic shifts in ecosystems, Nature, 413 (11), p. 591-596.

Steyaert P., Ollivier G., 2007. The European Water Framework Directive: how ecological assumptions frame technical and social change, Ecology and Society 12(1): 25. [En Ligne] : [http://www.ecologyandsociety.org/vol12/iss 1/art25/].
Stoddard J.L., Larsen D.P., Hawkins C.P., Johnson R.K., Norris R.H., 2006. Setting expectations for the ecological condition of streams: The concept of reference condition, Ecological Applications, 16, 4, p. 1267-1276.

Verdonschot P. F. M., Spears B. M., Feld C. K., Brucet S., Keizer-Vlek H., Borja A., Elliott M., Kernan M., JohnSon R. K., 2013. A comparative review of recovery processes in rivers, lakes, estuarine and coastal waters, Hydrobiologia, 704(1), p. 453-474.

Wallace K.J., 2007. Classification of ecosystem services : problems and solutions. Biological Conservation, 139, p. 235246.

WFD CIS (2010). Guidance document on the intercalibration process 2008-2011. Working Group ECOSTAT Guidance Document $n^{\circ} 14,19$ p. + annexes. 